94 LOWRY, PICKARD, AND KENYON.: THE ROTATORY DISPERSIVE

\title{
XII. - The Rotatory Dispersive Power of Organic Compounds. Part V. A Comparison of the Optical and Magnetic Rotatory Dispersions in Some Optically Active Liquids.
}

\section{By Thomas Martin Lowry, Robert Howson Pickard, and Joseph KenYon.}

ONE of the first objects aimed at in the present experiments on the rotatory dispersive power of organic compounds was to compare (1) the natural rotatory disperson and (2) the magnetic rotatory dispersion in a number of optically active liquids. The substances which have been available hitherto for making such a comparison have usually been of complicated structure, as in the case of pinene and nicotine; or they have been difficult to prepare in a state of optical purity, as in the case of turpentine and optically active amyl alcohol; they may even have shown anomalous rotatory dispersion, as in the case of ethyl tartrate. The preparation of a large number of active alcohols in a state of optical purity (see 
Pickard and Kenyon, T., 1911, 99, 45 ; 1912, 101, 620 ; 1913, 103, 1923 ; and P., 1912, 28, 42) provided a unique supply of material for the comparison referred to above, and led directly to the co-operation in experimental work which forms the basis of the present paper. The magnetic rotations and dispersions in most of the compounds used in this investigation have been given in Part IV of this series of papers; the corresponding optical rotations and dispersions are set out in the following pages.

\section{Wiedemann's Law.}

The comparison of the optical and magnetic rotatory dispersions in an optically active liquid was first made by G. Wiedemann in 1851, five years after the "magnetisation of light" had been alscovered by Faraday. Wiedemann made a series of comparative measurements in the case of turpentine oil with solar light of five different wave-lengths. After working out the ratio of the two rotations for each wave-length, he concluded that "These numbers agree so well together that one may assume that the law of proportionality of the rotation of the plane of polarisation produced by the current in light of different wave-lengths, with the rotation already existing in turpentine-oil may be regarded as correct" (Ann. Phys. Chem., 1851, [ii], 82, 231).

After an interval of fifty years Wiedemann's law was tested by Disch (Ann. Physik, 1903, [iv], 12, 1153), who discovered marked deviations, especially in substances showing anomalous rotatory dispersion, but concluded that these were due to lack of homogeneity in the material. Darmois (Ann. Chim. Phys., 1911, [vii], 22, 247, 495), from very similar data, concluded that "the law of proportionality was quite inexact, and that Wiedemann's result was the result of a pure chance."

A series of experiments on quartz (Lowry, Phil. Trans., 1912, A, 212,295 ) showed that in this case the proportionality between the natural and artificial rotatory powers was exact within the limits of experimental error over a wide range of the visible spectrum. The experiments now described show that this proportionality, which is perhaps a general property of optically active crystals, does not exist in the case of optically active liquids. Occasionally, as in the case of phenylmethylcarbinol, the optical and magnetic dispersion-ratios come very close together, but the accidental character of this agreement is revealed by the wide disagreement in the dispersion-ratios $\alpha_{4359} / \alpha_{5461}$ of the next homologue, thus :

$$
\left\{\begin{array}{ccc}
\mathrm{C}_{6} \mathrm{H}_{5} \cdot \mathrm{CH}(\mathrm{OH}){ }^{\bullet} \mathrm{CH}_{3} \text { (opt.) } \ldots . . . & 1 \cdot 736 \\
\text { (mag.) } \ldots & 1.739
\end{array}\right\} \quad\left\{\begin{array}{cc}
\mathrm{C}_{6} \mathrm{H}_{5} \cdot \mathrm{CH}(\mathrm{OH}) \cdot \mathrm{C}_{4} \mathrm{H}_{5} \text { (opt.). } & 1 \cdot 674 \\
\text { (mag.). } & 1 \cdot 731
\end{array}\right\}
$$


Equally emphatic evidence that Wiedemann's law cannot be applied to organic liquids is afforded by the behaviour of the series of fatty alcohols. Thus we find that the characteristic dispersionratios for the following series of carbinols are:

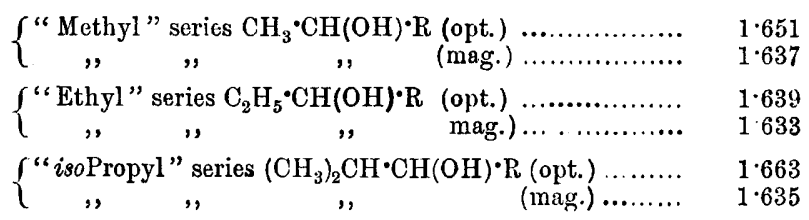

The lowest members of the series to show optical activity contain at least four carbon atoms; their optical rotatory dispersions show marked anomalies (p. 84), but their magnetic dispersions are perfectly normal, thus proving again that Wiedemann's law cannot be applied to them.

In the case of more complex compounds the discrepancy is still larger. Thus, in the case of a complex ester, there is the following remarkable contrast between the two dispersion-ratios:

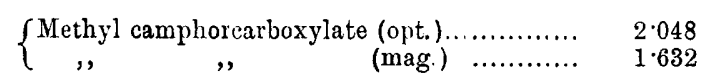

Ethyl tartrate, which shows anomalous optical rotatory dispersion, agrees with this substance in giving a magnetic dispersion-ratio $1 \cdot 630$, which does not differ trom the ratio characteristic of such simple esters as ethyl acetate.

It is not easy to explain this inequality in dispersive power. In seeking for an explanation the chief clue is to be found in the fact that the difference between the optical and magnetic dispersions vanishes in the case of quartz, where the optical activity is due to asymmetry in the crystal structure instead of in the molecule. In this case it may be suggested that every part of the molecule contributes its quota both to the optical and to the magnetic rotatory power. In optically active liquids, on the other hand, it is probable that the natural rotatory power is influenced to a much greater degree by the atoms or groups of atoms which are nearest to the centres of asymmetry, whilst the magnetic rotatory power is influenced to an equal extent by all atoms of a given kind. The existence of a rough "additive law" for magnetic rotations and the highly constitutive character of optical rotatory power may thus perhaps account for the inequality of the two dispersions.

The deviations from Wiedemann's law in the case of optically active liquids suggest a further question, to which at the present time no decisive answer can be given. The inequality in the two dispersions might be attributed: (a) to a change in the value of the 
"dispersion-constant," $\lambda_{0}{ }^{2}$, in the equation $a=\frac{k_{0}}{\lambda^{2}-\lambda_{0}{ }^{2}}$ (see part II), or $(b)$ to the introduction (as in the case of ethyl tartrate) of a second term into the dispersion-equation, which then becomes:

$$
a=\frac{k_{0}}{\lambda^{2}-\lambda_{0}^{2}}+\frac{k_{1}}{\lambda^{2}-\lambda_{1}^{2}} \text {. }
$$

In the case of ethyl tartrate, the second term in the equation for the optical rotation is negative in sign; it is therefore easy to detect its influence in the anomalous rotatory dispersion of the substance (Lowry and Dickson, P., 1913, 29, 185). No anomaly exists, however, in the magnetic rotatory dispersion of this ester, and no deviation from the normal form of the dispersion-curve can be detected, in spite of the great probability that the liquid contains two dynamic isomerides with independent dispersion-constants. It is therefore evidently very difficult to detect the presence of a second term in the dispersion-equation unless the two terms differ in sign or contain dispersion-constants differing very widely in magnitude.

From the theoretical point of view much might be said in favour of adopting the second of the explanations set out above, but in actual practice no such option exists. There is, in fact, no alternative to the employment of the simple formula which assumes that the deviations in Wiedemann's law are due to changes in the value of the dispersion-constant $\lambda_{0}{ }^{2}$. This formula, as has been shown in Part II of this series of papers, expresses the form of the dispersion-curves, both for optical and for magnetic rotations, with an accuracy which exceeds that which can be attained in any one individual series of observations; it would therefore be quite impossible to determine the magnitude of the four constants in the two-term equation, even if it were known that this equation was the correct one to apply.

\section{Optical Rotatory Dispersion in Homologous Series.}

There is a marked tendency for the optical (like the magnetic) rotatory dispersions in homologous series of compounds to settle down to a steady value after the first members have been passed; but the steady value does not appear until the ecmpound contains five or six carbon atoms, the abnormalities usually persisting until the growing chain has established itself as the largest of the four groups attached to the asymmetric carbon atom. Thus we have: 


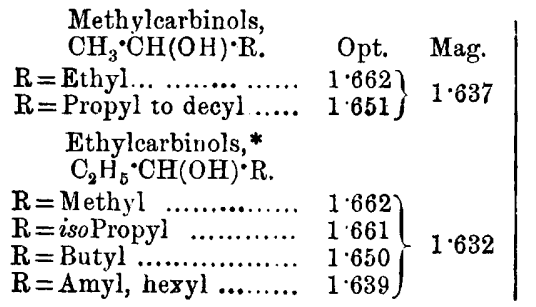

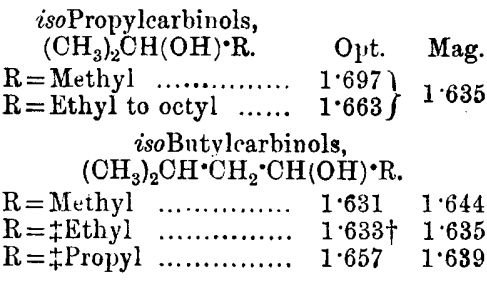

* The diethylcarbinol is, of course, inactive; the ethylpropylcarbinol has too small a rotatory power for an exact measurement of the dispersion ratio.

$\dagger$ The agreement with the magnetic dispersion ratio 1.685 is exceptionally close, but does not reappear in the two adjacent homologues and must therefore be regarded as fortuitous.

¥ These samples were probably not quite pure, but this would not be likely to affect the dispersion-ratios.

The dispersive power of methylisopropylcarbinol is remarkable, especially in contrast with the steady value 1.663 of the dispersionratio in six higher homologues. It is. however, fully justified by a consideration of the dispersion-ratios in the series set out below:

\begin{tabular}{|c|c|}
\hline 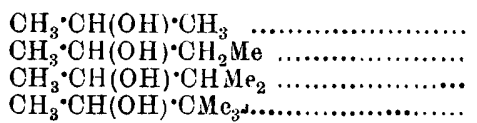 & $\begin{array}{l}\text { inactive } \\
1 \cdot 662 \\
1 \cdot 697 \\
1 \cdot 707\end{array}$ \\
\hline
\end{tabular}

Attention may also be directed to the high dispersive power of :

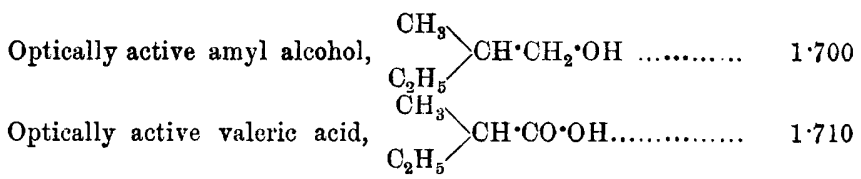

These two compounds are very similar in structure, and differ from the active secondary alcohols in that the asymmetric carbon atoms are linked entirely to carbon and hydrogen, instead of to carbon, hydrogen, and oxygen. The fact that the oxygen has been shifted away from the asymmetric atom, instead of diminishing the rotatory dispersion, actually ircreases it. It appears, in fact, that oxygen contributes relatively little to the rotatory dispersion of these compounds, which seems to be influenced more by carbon than it is by oxygen.

\section{Absolute Molecular Rotation (Optical).}

In dealing with the magnetic rotatory power of liquids, Perkin selected water as the standard both of specific and of molecular rotation. This choice was justifiad by the fact that it is much easier to measure the rotation produced in a decimetre length of 
water than to determine the strength of the magnetic field which produces this effect. In dealing with the natural rotatory power of optically active liquids, no such standard is needed, as this property can be measured directly in angular degrees per decimetre length of the liquid; a correction for density or concentration gives the "specific rotation," from which the "molecular rotation" may easily be calculated without introducing any other substance as a basis for comparison.

This fundamental difference in the method of dealing with natural and magnetic rotations leads to a further contrast when the attempt is made to eliminate the influence of dispersion by working out the "absolute" rotatory powers of a substance. In the case of magnetic rotatory powers the comparative method was extended to this final stage in the working out of the experimental observations, the magnetic rotation in water being reduced to "absolute" wave-length as well as that in the substance. In consequence of this extension of the comparative method the final reduction produces only slight alterations in the values of the molecular rotatory powers; in the case of substances, such as tert.-butyl alcohol, which have the same dispersive-power as water, the "absolute" molecular rotation is actually identical with that for sodium or for mercury light; a table showing the small changes produced in the case of typical aliphatic compounds is given in Part IV (this vol., p. 88). In dealing with optical rotatory powers, it is necessary, in order to eliminate the influence of dispersion, to reduce the readings for the substance to the standard wave-length, at which $\lambda^{2}=1+\lambda_{0}^{2}$. This produces large alterations, which cannot be diminished or removed by the use of relative values for two substances. The actual alterations which are produced are hown in the following table for a series of typical dispersion-ratios:

and

$\begin{array}{llllll}a_{4359} / \alpha_{5461}=1.636 & 1.644 & 3.651 & 1.673 & 1735 & 1.764 \\ a_{\text {abs. }} / \alpha_{5461}=0.2784 & 0.2763 & 0.2745 & 0.2691 & 0.2555 & 0.2499 \\ a_{\text {abs. }} / \alpha_{5893}=0.3275 & 0.3254 & 0.3237 & 0.3181 & 0.3045 & 0.2989\end{array}$

In this table it is seen that the absolute rotations range from 28 to 25 per cent. of the rotations for green mercury light, and from 33 to 30 per cent. of the rotations for sodium light.

\section{Tabulated Measurements.}

In table I two series of values are given for the absolute molecular rotation of each compound. The values given in the 


\section{LOWRY, PICKARD, AND KENYON: THE ROTATORY DISPERSIVE}

fifth column are calculated from the readings of column 4, which were made in London with green mercury light; the values in the sixth column are calculated from readings which were made in Blackburn with sodium light. In nearly every case the two values agree together closely, but in the few cases in which marked differences are observed, the second value is to be regarded as the more trustworthy. Thus in the case of methylethylcarbinol, the lower value obtained with mercury light is almost certainly due to the absorption of water, as it was found to diminish steadily in successive series of experiments; so also in the case of methyl$n$-butylcarbinol, the sample used for measuring the dispersion was known to be of slightly lower rotatory power than the sample used when the sodium readings were taken.

The dispersion-ratios and dispersion-constants are set out in columns 2 and 3 . The rotatory dispersion in these compounds is so steady that no new facts are disclosed when the effects of dispersion are eliminated; all the essential characteristics of the drift of rotatory power in honologous series can be seen equally well in the readings for sodium or for mercury light. Attention may, however, be directed to the fact that the small rotations in the "ethyl"-carbinols are associated with exceptionally small dispersion, whilst the high optical activity of the "isopropyl "-carbinols is associated with a high dispersive power; the "methyl"-carbinols occupy an intermediate position both in rotatory power and in dispersive power. This parallelism between rotation and dispersion does not apply to the abnormality of the initial members of each series, where a low rotatory power may be associated with high dispersive power.

Optical Rotatory Dispersion and Absolute Molecular Rotation in some Optically Active Liquids.

\begin{tabular}{|c|c|c|}
\hline & Dispersive & $\begin{array}{l}\text { Observed } \\
\text { rotation, }\end{array}$ \\
\hline & & $\begin{array}{c}a_{5461} \\
(100 \mathrm{~mm} .) .\end{array}$ \\
\hline
\end{tabular}

(a) "Methyl" Carbinols (normal).

\begin{tabular}{|c|c|c|c|c|c|c|c|c|}
\hline Methy & ethyl c & binol & & $1 \cdot 661$ & 262 & $12.57^{\circ}$ & {$[3 \cdot 13]$} & $3 \cdot 30$ \\
\hline , & jropyl &, & $\ldots \ldots \ldots$ & 1.652 & $2 \cdot 40$ & $13 \cdot 25$ & 3.95 & $3 \cdot 91$ \\
\hline ," & butyl & $"$ & ........... & 1.653 & $2 \cdot 42$ & $10 \cdot 84$ & {$[3 \cdot 72]$} & $3 \cdot 81$ \\
\hline ,, & amyl & ,", & $\ldots \ldots$ & $1 \cdot 651$ & $2 \cdot 37$ & $9 \cdot 88$ & $3 \cdot 85$ & $3 \cdot 88$ \\
\hline ,, & hexyl & ," & $\ldots \ldots \ldots$ & $1 \cdot 653$ & $2 \cdot 42$ & $9 \cdot 54$ & $4 \cdot 14$ & $4 \cdot 10$ \\
\hline ," & heptyl & ,, & . & $1 \cdot 651$ & $2 \cdot 37$ & $8 \cdot 68$ & $4 \cdot 17$ & 7 \\
\hline ,. & octyl & , & .......... & $1 \cdot 649$ & $2 \cdot 32$ & $8 \cdot 44$ & $4 \cdot 45$ & $\cdot 43$ \\
\hline ,, & nonyl & $"$ & ......... & $1 \cdot 651$ & $2 \cdot 37$ & $7 \cdot 87$ & 450 & 4 \\
\hline ,", & decyl & ", & . ....... & $1 \cdot 653$ & $2 \cdot 42$ & $7 \cdot 52$ & $4 \cdot 61$ & \\
\hline
\end{tabular}


POWER OF ORGANIC COMPOUNDS. PART V.

Optical Rotatory Dispersion and Absolute Molecular Rotation in some Optically Active Liquids (continued).

Observed

Dispersive rotation,

Dispersion - power, $\boldsymbol{\alpha}_{5461}$ ratio. $100 \lambda_{0}^{2}$. (100 mm.). L. F. \& K.

(b) "Ethyl" Carbinols.

\begin{tabular}{|c|c|c|c|c|c|c|c|c|}
\hline Ethyl & propyl & binol & ........... & {$[1 \cdot 615 j *$} & {$[1 \cdot 39]^{*}$} & $1 \cdot 60^{\circ}$ & 057 & 0.67 \\
\hline ," & butyl & ,, & .......... & 1.650 & $2 \cdot 34$ & $7 \cdot 94$ & $3 \cdot 09$ & $3 \cdot 13$ \\
\hline ", & amyl & ," & ......... & $1 \cdot 639$ & $2 \cdot 05$ & $8 \cdot 07$ & $3 \cdot 54$ & $3 \cdot 50$ \\
\hline ," & hexyl & ,", & & $1 \cdot 639$ & $2 \cdot 05$ & $7 \cdot 80$ & $3 \cdot 78$ & $3 \cdot 78$ \\
\hline 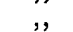 & octyl & ," & ......... & $1 \cdot 634$ & 192 & 6.14 & $3 \cdot 56$ & $3 \cdot 55$ \\
\hline
\end{tabular}

(c) "isoPropyl" Carbinols.

\begin{tabular}{|c|c|c|c|c|c|c|c|c|}
\hline isoPropyl & methyl & rrbinol & & $1 \cdot 697$ & $3 \cdot 46$ & $4 \cdot 74^{\circ}$ & $1 \cdot 34$ & 1.34 \\
\hline, & ethyl & ,, & $\ldots$ & $1 \cdot 661$ & $2 \cdot 62$ & $14 \cdot 71$ & $4 \cdot 95$ & $4 \cdot 94$ \\
\hline ", & propyl & ," & $\ldots$ & $1 \cdot 665$ & $2 \cdot 74$ & $20 \cdot 62$ & $7 \cdot 89$ & 789 \\
\hline ", & butyl & ", & ... & $1 \cdot 665$ & $2 \cdot 74$ & $24 \cdot 97$ & $10 \cdot 69$ & $10 \cdot 64$ \\
\hline ," & amyl & ," & ... & $1 \cdot 663$ & $2 \cdot 67$ & $22 \cdot 46$ & $10 \cdot 63$ & 10.53 \\
\hline ," & hexyl & , & $\ldots$ & $1 \cdot 661$ & $2 \cdot 62$ & $21 \cdot 16$ & 11.01 & $10 \cdot 86$ \\
\hline ", & octyl &, & $\ldots$ & 1.661 & $2 \cdot 62$ & $18 \cdot 38$ & $11 \cdot 13$ & $11 \cdot 06$ \\
\hline ", & decyl & ," & $\ldots$ & 1.669 & $2 \cdot 86$ & $15 \cdot 59$ & 10.82 & $11 \cdot 06$ \\
\hline
\end{tabular}

(d) "Butyl" Carbinols.

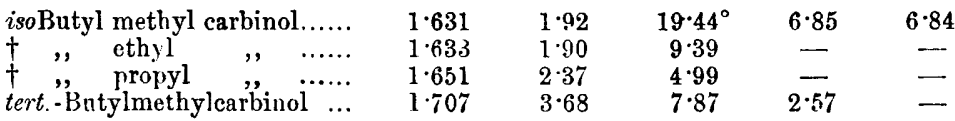

(e) Aromatic Secondary Alcohols.

\begin{tabular}{|c|c|c|c|c|c|}
\hline Phenylmethylcarbinol $\quad . . . .$. & $1 \cdot 736$ & $4 \cdot 29$ & $52 \cdot 49^{\circ}$ & $16 \cdot 14$ & $\begin{array}{l}15 \cdot 93 \\
11 \cdot 98\end{array}$ \\
\hline Phenylethylcarbinol & 1.674 & 2.93 & $32 \cdot 37$ & $11 \cdot 91$ & $11 \cdot 98$ \\
\hline Benzylmethylcarbinol .......... & $1 \cdot 833$ & $6 \cdot 13$ & $32 \cdot 47$ & $10 \cdot 57$ & - \\
\hline $\boldsymbol{\beta}$-Phenylethylmethylcarbinol & $1 \cdot 679$ & 3.03 & 16.55 & $6 \cdot 79$ & - \\
\hline $\begin{array}{l}\text { Amyl alcohol (active) ... } \\
\text { iso Valeric acid ............... }\end{array}$ & $\begin{array}{l}1 \cdot 700 \\
1 \cdot 710\end{array}$ & $\begin{array}{l}3 \cdot 54 \\
3 \cdot 74\end{array}$ & $\begin{array}{c}5 \cdot 40^{\circ} \\
20 \cdot 50\end{array}$ & $\overline{5 \cdot 80}$ & - \\
\hline
\end{tabular}

* 'The rotatory power was too small to give trustworthy figures for the dispersive power of this compound.

+ These samples were of doubtful purity.

\section{Summary and Conclusions.}

1. Optical rotatory dispersion varies more widely than magnetic rotatory dispersion, but remains constant in homologous series of secondary aliphatic alcohols when the "growing chain" of carbon atoms has established itself as the heaviest radicle in the asymmetric molecule.

2. Wiedemann's law, which applies exactly in the case of quartz, does not hold good in the case of optically active liquids. The optical is usually greater than the magnetic rotatory dispersion, but the converse is sometimes observed in aromatic compounds. 
3. Remarkable variations of optical rotatory dispersion are observed in the series:

$$
\begin{aligned}
& \mathrm{C}_{6} \mathrm{H}_{5} \cdot \mathrm{CH}(\mathrm{OH}) \cdot \mathrm{CH}_{3} \ldots \ldots \ldots \ldots \ldots . . \quad \alpha_{4359} / \alpha_{5461}=1 \cdot 736 \\
& \mathrm{O}_{6} \mathrm{H}_{5} \cdot \mathrm{CH}(\mathrm{OH}) \cdot \mathrm{CH}_{2} \cdot \mathrm{CH}_{3} \ldots \ldots \ldots \ldots, \quad, \quad=1 \cdot 674 \\
& \mathrm{C}_{6} \mathrm{H}_{5} \cdot \mathrm{CH}_{2} \cdot \mathrm{CH}(\mathrm{OH})^{\circ} \mathrm{CH}_{3} \ldots \ldots \ldots \ldots . . \quad, \quad, \quad=1.833 \\
& \mathrm{C}_{6} \mathrm{H}_{5} \cdot \mathrm{CH}_{2}^{2} \cdot \mathrm{CH}_{2} \cdot \mathrm{CH}(\mathrm{OH}) \cdot \mathrm{CH}_{3} \ldots \ldots . ., \quad, \quad=1 \cdot 679
\end{aligned}
$$

and in the series:

$$
\begin{aligned}
& \mathrm{CH}_{3} \cdot \mathrm{CH}(\mathrm{OH}) \cdot \mathrm{CH}_{2} \mathrm{Me} \ldots \ldots \ldots \ldots \ldots, \quad, \quad=1.662 \\
& \mathrm{CH}_{3} \cdot \mathrm{CH}(\mathrm{OH}) \cdot \mathrm{CHMe} \mathrm{Me}_{2} \ldots \ldots \ldots \ldots \ldots ., \quad, \quad=1.697 \\
& \mathrm{CH}_{3} \cdot \mathrm{CH}(\mathrm{OH}) \cdot \mathrm{CMe}_{3} \ldots \ldots \ldots \ldots \ldots \ldots . . . \quad, \quad=1.707
\end{aligned}
$$

High dispersions are also observed in:<smiles>CCC(C)C(C)C(C)C(=O)O</smiles>

The expenses incurred in the researches described in this and in the preceding paper have been defrayed in part by generous grants from the Government Grant Committee of the Royal Society, which are hereby gratefully acknowledged. Our thanks are also due to Mr. W. P. Paddison, to Mr. H. W. Southgate, and to Mr. H. R. Courtman for assistance in carrying out the long and tedious series of observations recorded in these papers.

Guy's Hospital.

LoNDON, S.E.
Municipal Techical School.

BLACKBURN. 